\title{
Étude de la résistance au chlorpyrifos à partir de quelques souches du moustique Culex pipiens $\mathrm{L}$. du sud de la France
}

\author{
M. RAYMOND*, B. GAVEN **, Nicole PASTEUR * et G. SINEGRE** \\ * Institut des Sciences de l'Evolution, Laboratoire de Génétique \\ Université de Montpellier II, place Eugène-Bataillon, F 34060 Montpellier \\ ** Entente Interdépartementale pour ia Démoustication \\ avenue Rimbaud, B.P. 6036, F 34030 Montpellier
}

\begin{abstract}
Résumé
Une population de Culex pipiens, prélevée en 1979 au sein d'une zone régulièrement traitée, a été soumise en laboratoire pendant plus de 30 générations à différents régimes d'élevage (en exerçant ou non une pression de sélection au chlorpyrifos). L'analyse des différentes souches issues de cette population a permis de montrer : 1) qu'un ou plusieurs nouveaux facteurs de résistance, différents de l'allèle Est-3A déjà identifié depuis 1975, sont présents et 2) que l'allèle Est - $^{A}$, ou un gène qui lui est associé, induit un retard dans le développement larvaire quand la pression de sélection est relâchée. La nature du ou des facteurs de résistance est discutée, ainsi que ses interactions avec l'allèle Est-3A.

Mots clés : Sélection, organophosphates, estérases, Culex pipiens.
\end{abstract}

\begin{abstract}
Summary
Chlorpyrifos resistance in various Culex pipiens L. mosquito strains from the South of France
\end{abstract}

A wild population of Culex pipiens was captured in 1979 from a regularly treated area, and reared with or without chlorpyrifos selection for more than 30 generations. The analysis of different strains derived from this population showed : 1) that one or several new resistance factors, different from the Est-3A allele identified in 1975, have been selected, and 2), that the Est-3A allele or a gene associated with it induces a longer larval development when the selection pressure is relaxed. The nature of the resistance factors and its interactions with the Est-3A allele are discussed.

Key words : Selection, organophosphates, esterases, Culex pipiens.

\section{Introduction}

Les populations de Culex pipiens L. sont soumises depuis 1968 à un contrôle intense par des insecticides essentiellement organophosphorés (chlorpyrifos jusqu'en 1981) dans le Languedoc-Roussillon. 
Dès 1972, SINEgre et al. (1976) enregistraient une baisse de sensibilité au chlorpyrifos dans les environs de Lunel (Hérault). En 1974-1975, la résistance avait considérablement augmenté et s'étendait le long du littoral languedocien. Les travaux de PAsteur \& Sinegre $(1975,1978$ a) sur des populations naturelles et de Pasteur (1977), Pasteur \& Sinegre (1978 b) et Pasteur et al. (1981 a) sur une souche (S54 ou S5) isolée en 1975 permettent de déduire que le principal facteur de résistance, présent à cette époque dans les populations naturelles de Culex pipiens, était une estérase de détoxification à grande activité nommée Estérase-3 codée par un gène (Est-3). Le gène Est-3 possède 2 allèles : un allèle dominant, Est-3A, codant l'Estérase-3 ayant une grande activité et un allèle "nul » récessif, Est-3O; 98 p. 100 des individus homozygotes pour l'allèle Est-3 ${ }^{A}$ sont tués par des concentrations de chlorpyrifos de $0,02 \mathrm{mg} / 1$ (ppm), alors que la même mortalité est provoquée par $0,0008 \mathrm{mg} / \mathrm{l}$ de chlorpyrifos dans les souches sensibles ne possédant pas l'allèle Est-3A.

En 1977, Sinegre et al. observaient, dans la région de Maurin, la présence de populations montrant une mortalité d'environ 60 p. 100 après une exposition à 0,02 ppm de chlorpyrifos. En 1979, ces chercheurs réussissaient à maintenir au laboratoire une population "épigée " prélevée à Maurin. (Rappelons que les populations épigées de Culex pipiens qui colonisent des gîtes à ciel ouvert par opposition aux populations "hypogées " colonisant des gîtes souterrains - caves ou vides sanitaires sous les habitations - , sont généralement eurygames et ne se reproduisent que très difficilement dans les conditions de laboratoire.)

Nous étudions ici l'évolution de la résistance de la souche Maurin prélevée en 1979 et de la souche S54 prélevée en 1975 quand elles sont soumises à différentes pressions de sélection et quand cette sélection est relâchée. A titre de comparaison, une $3^{\text {c }}$ souche, Bleuet, isolée en 1961 par Rioux \& PECH (1961) à une époque où les insecticides organophosphorés n'étaient pas utilisés en France, a été également analysée et sert de souche sensible de référence.

\section{Matériel et méthodes}

Les souches Maurin et S54 sont maintenues en laboratoire dans des cages cubiques de $50 \mathrm{~cm}$ de côté. Les souches S54 et Bleuet sont autogènes, et leurs femelles se reproduisent sans repas de sang. La souche Maurin, provenant d'une population épigée, est anautogène, et l'est restée jusqu'au début de l'année 1983 (c'est-à-dire pendant 4 ans); il était donc nécessaire pour obtenir des pontes de fournir un repas de sang aux femelles en plaçant dans la cage un pigeon ou une souris.

Les œufs sont recueillis dans des godets de plastique où ils éclosent. Les jeunes larves sont alors transférées dans des bacs de $37 \mathrm{~cm} \times 22,5 \mathrm{~cm} \times 7 \mathrm{~cm}$ où elles sont nourries avec du biscuit de chien. Selon l'expérience en cours, les larves de $4^{\mathrm{e}}$ stade sont ou non exposées pendant $24 \mathrm{~h}$ à une concentration de chlorpyrifos provoquant une mortalité comprise entre 60 et 80 p. 100. Les nymphes issues des larves survivantes sont transférées dans la cage d'adultes où elles émergent. Chaque génération de chaque souche est issue d'au moins 500 individus de la génération précédente. 
Régulièrement, un test de sensibilité a été réalisé en exposant des lots de 20 larves mises dans $100 \mathrm{ml}$ d'eau contenant différentes concentrations de chlorpyrifos (dissous dans de l'alcool éthylique à $95^{\circ}$ ). Après $24 \mathrm{~h}$, les larves mortes ou moribondes sont dénombrées. Chaque test comprenant un témoin sans insecticide est répété environ 5 fois de façon à ce que, dans la mesure du possible, l'échantillon de larves exposées à chaque concentration d'insecticide soit au moins de 100 . Les résultats sont analysés sur un ordinateur TRS-80, RAYMOND, 1984 (résultats non publiẻs).

Quand cela était nécessaire, le phénotype d'Est-3 a été déterminé chez chaque individu soit par électrophorèse (PASTEUR, 1977), soit en utilisant une modification de la «technique du papier filtre » décrite par PAsteur \& Georghiou (1981).

\section{Résultats}

\section{A. Souche 554}

Cette souche est homozygote pour un facteur unique de résistance, $C h l^{R}$ (PASTEur \& SinEgre, 1978 b) et pour l'allèle Est-3A qui code l'Estérase-3 de grande activité (PASTEur, 1977). Génétiquement, les allèles $E s t-3^{A}$ et $C h l^{R}$ sont inséparables et Pasteur et al. (1981 b) ont montré que l'Estérase-3 hydrolyse le chlorpyrifos. De ces observations, on peut conclure que, dans la souche S54, la résistance au chlorpyrifos est due à l'allèle $E s t-3^{A}$ qui code une enzyme de détoxification de grande activité ; cet allèle est absent dans les souches sensibles comme Bleuet.

\section{TABLEAU 1}

Exemple de quelques mortalités observées dans la souche S54 au cours des générations d'élevage sans pression de sélection.

Example of some mortalities observed in the 554 strain raised without chlorpyrifos selection.

\begin{tabular}{|c|c|c|c|c|c|}
\hline \multirow{2}{*}{ Générations } & \multicolumn{3}{|c|}{ Mortalités (\%) aux doses (ppm) } & \multirow{2}{*}{ DL 50} & \multirow{2}{*}{ DL 90} \\
\hline & 0,0064 & 0,0128 & 0,0256 & & \\
\hline $\mathrm{F} 2 \ldots \ldots \ldots$ & 0 & 90 & 100 & 0,0075 & 0,012 \\
\hline F3 $\ldots \ldots \ldots$ & 0 & 84 & 100 & 0,011 & 0,015 \\
\hline F4 $\ldots \ldots \ldots$ & 1 & 94 & 100 & 0,010 & 0,012 \\
\hline F5 $\ldots \ldots \ldots$ & 0 & 93 & 100 & 0,010 & 0,012 \\
\hline F6 .. & 2 & 86 & 100 & 0,010 & 0.013 \\
\hline F15 . & 0 & 26 & 100 & 0,015 & 0,02 \\
\hline F30 & 0 & 70 & 100 & 0,012 & 0,015 \\
\hline F31 & 0 & 52 & 100 & 0,013 & 0,016 \\
\hline F33 & 0 & 38 & 100 & 0,014 & 0,019 \\
\hline F34 & 0 & 66 & 100 & 0,012 & 0,016 \\
\hline F35 & 0 & 60 & 100 & 0,011 & 0,016 \\
\hline F42 & 0 & 89 & 100 & 0,011 & 0,013 \\
\hline F55 & 2 & 70 & 100 & 0,011 & 0,015 \\
\hline F60 & 2 & 30 & 100 & 0,014 & 0,017 \\
\hline
\end{tabular}

Aucune perte de résistance n'est observée.

No decrease in resistance is observed. 
1. Évolution de la résistance en l'absence de pression de sélection

La souche S54 est maintenue au laboratoire sans pression de sélection depuis son isolement en 1975. La $\mathrm{DL}_{\overline{5}(\text { ) }}$ est d'environ $0,011 \mathrm{ppm}$, et n'a pratiquement pas varié depuis huit ans (tabl. 1). Nous avons contrôlé que la souche S54 était toujours probablement homozygote pour l'allèle Est-3A : sur 249 individus examinés, tous possédaient cette enzyme.

\section{Tableau 2}

Mortalités observées chez les larves de la souche S54, soumises à 0,01 ppm de chlorpyrifos pendant 12 générations successives.

Mortalities observed among larvae of the S54 strain exposed each generation to $0.01 \mathrm{ppm}$ chlorpyrifos.

\begin{tabular}{|c|c|c|c|}
\hline Génération & Larves mortes & Total & Mortalité (\%) \\
\hline$\ldots \ldots \ldots \ldots$ & 341 & 1000 & 34,1 \\
\hline F15 $\ldots \ldots \ldots \ldots$ & 369 & 1000 & 36,9 \\
\hline$F 16 \ldots \ldots \ldots \ldots$ & 416 & 1000 & 41,6 \\
\hline $\mathrm{F} 17 \ldots \ldots \ldots \ldots$ & 382 & 1000 & 38,2 \\
\hline F18 & 368 & 1000 & 36,8 \\
\hline F19 & 105 & 1000 & 10,6 \\
\hline$\ldots \ldots \ldots \ldots$ & 566 & 950 & 58,5 \\
\hline $\mathrm{F} 21 \ldots \ldots \ldots \ldots$ & 345 & 1000 & 34,5 \\
\hline F22 $\ldots \ldots \ldots \ldots \ldots$ & 209 & 995 & 21,0 \\
\hline F23 & 248 & 990 & 25,0 \\
\hline F24 & 351 & 996 & 35,2 \\
\hline F25 & 402 & 1000 & 40,2 \\
\hline
\end{tabular}

Les mortalités de la $14^{\circ}$ et $24^{\circ}$ générations ne sont pas significativement différentes. Mortalities observed in the $14 \mathrm{th}$ and $24 \mathrm{th}$ generations are not significantly different.

2. Évolution de la résistance en pression de sélection

Le tableau 2 donne les mortalités observées dans une souche dérivée de S54 maintenue pendant 12 générations en exposant à chaque génération des larves à $0,01 \mathrm{ppm}$ de chlorpyrifos (provoquant un peu moins de 50 p. 100 de mortalité). On ne constate aucune augmentation de résistance.

En conclusion, la résistance due à l'allèle Est-3A est stable dans la souche S54 que l'on relâche la sélection ou qu'on la maintienne.

\section{B. Souches issues de la population naturelle prélevée à Maurin}

Plusieurs souches ont été fondées à partir de la population naturelle prélevée à Maurin en 1979 (fig. 1). Dès son arrivée au laboratoire, cette population a été scindée en 2 lots. Le $1^{\text {er }}$ a été maintenu sans pression de sélection et a donné la souche Maurin-A. Le $2^{\text {nd }}$ a été élcvé en exposant les larves à des concentrations de chlorpyrifos induisant 60-80 p. 100 de mortalité à chaque génération, donnant ainsi la souche 
Maurin-B. A partir de la $34^{e}$ génération, la souche Maurin-B a de nouveau été scindée en 2 lots, l'un Maurin-B sur lequel la sélection a été poursuivie, l'autre Maurin-C sur lequel la sélection a été relâchée. Enfin, une dernière souche MSE a été isolée à partir de la descendance de couples de la $3^{\circ}$ génération de Maurin-C ne possédant pas l'Estérase-3 de grande activité.

(\% mortalité)

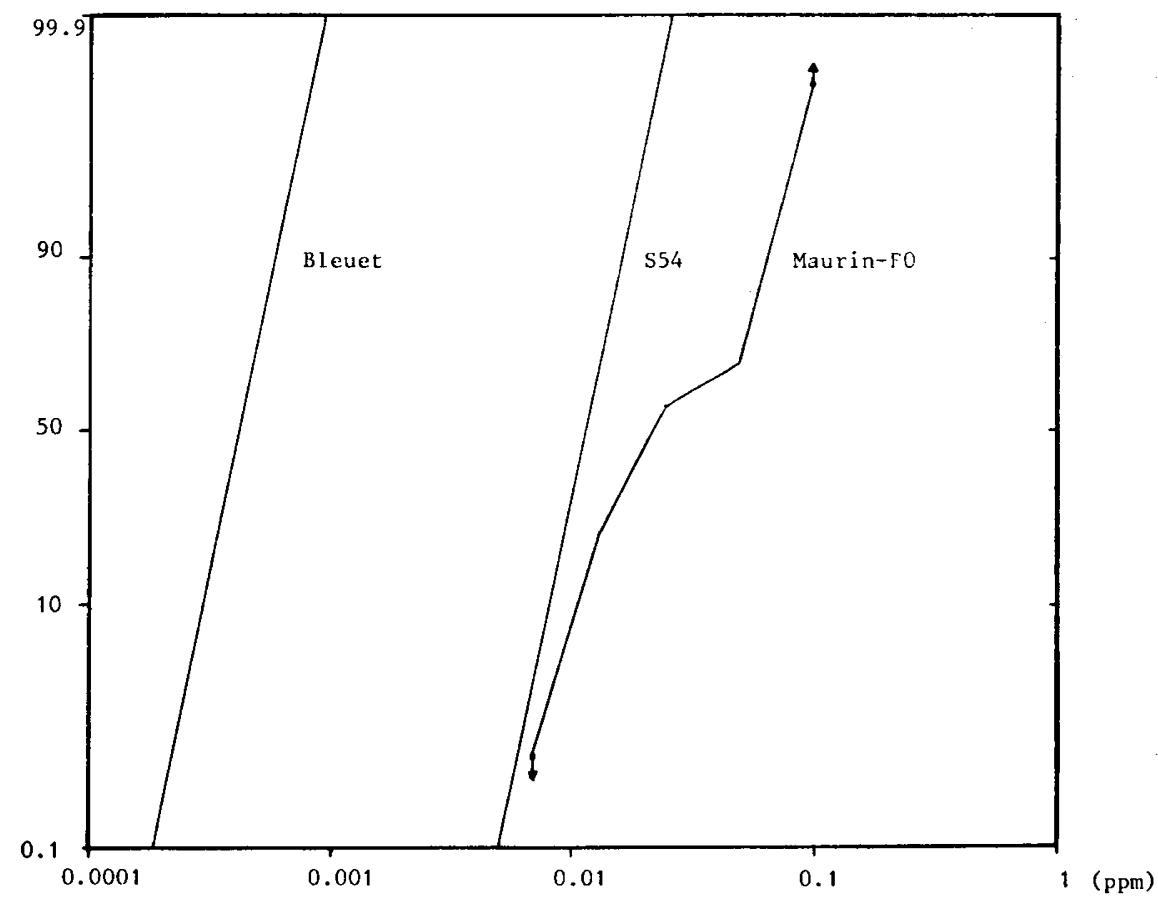

FIG. 1

Courbes de mortalité de la population naturelle Maurin-F0, prélevée en 1979, de la souche S54 (homozygote pour l'allèle Est-3A) et de la souche sensible Bleuet.

Mortality curves in the Maurin-F0 natural population captured in 1979, of the S54 strain (homozygous for the Est-3A allele) and of the Bleuet susceptible strain.

Chaque souche a été fondée à partir d'au moins 500 individus, ce qui nous permet de conclure que les événements que nous décrivons ci-dessous ne peuvent pas être imputables à des phénomènes d'effet fondateur. Seule la souche MSE a été fondée à partir de la descendance de 8 couples. 
1. Résistance de la population naturelle prélevée à Maurin (Fo des souches Maurin-A et Maurin-B)

La résistance observée dans cette population est supérieure à celle de la souche S54 : 45 p. 100 des larves meurent après une exposition à $0,02 \mathrm{ppm}$ de chlorpyrifos alors que 98 p. 100 des larves de S54 sont tuées par cette concentration (fig. 1). La $\mathrm{DL}_{50}$ était donc supérieure à $0,02 \mathrm{ppm}$ de chlorpyrifos. La courbe de mortalité de cette population n'est pas linéaire $(\mathrm{P}<0,0001)$ et présente un plateau à environ 65 p. 100 de mortalité. On peut donc penser que la population prélevée à Maurin n'était pas homogène quant à sa résistance. Nous ne possédons pas de données sur la fréquence de l'allèle Est-3A dans cette population au moment de son prélèvement. Toutefois, cette fréquence devait être élevée car 97 des 101 larves prélevées dans le même gîte en 1978 le possédaient (population 15 de PASTEUR et al., 1981 a).

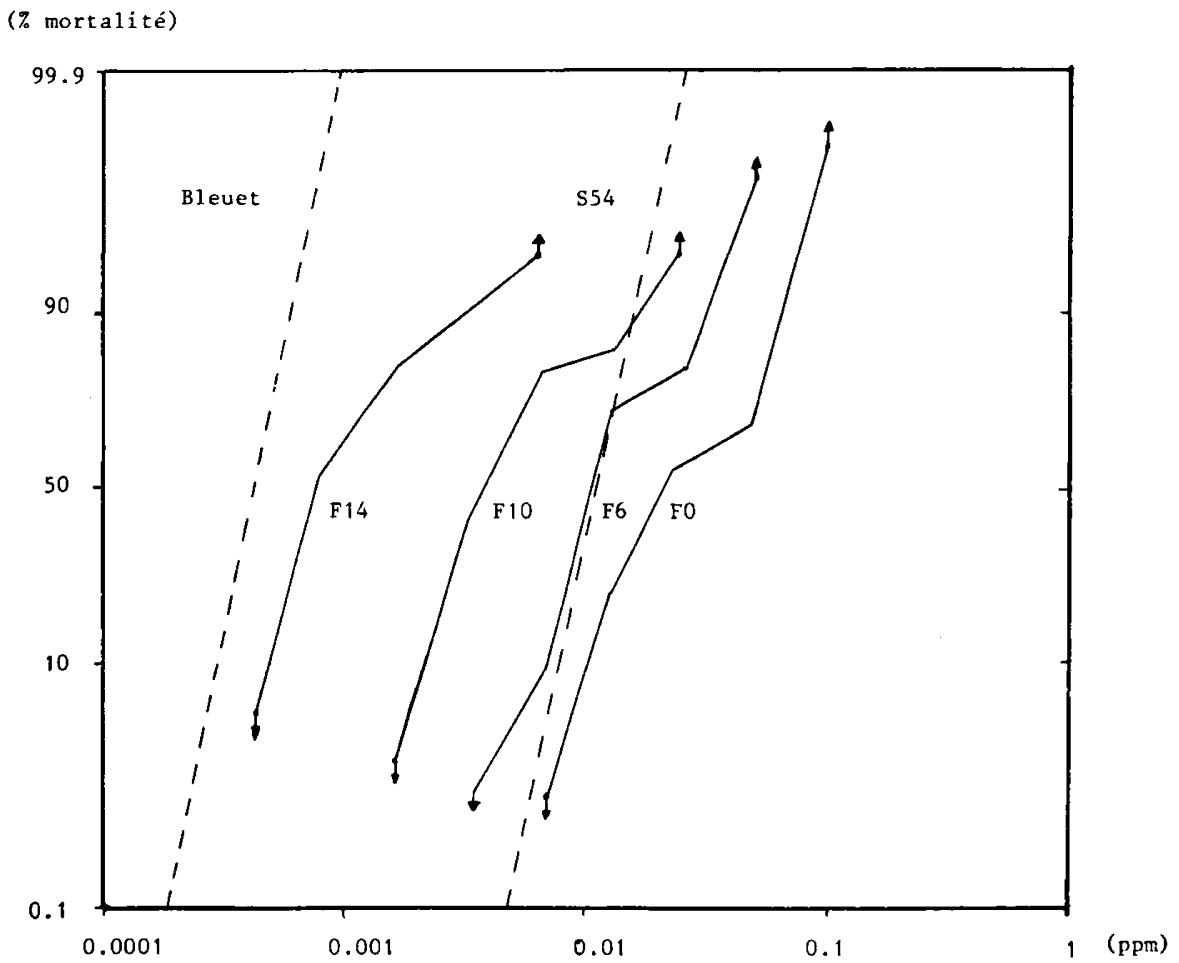

FIG. 2

Courbes de mortalité de quelques générations de la souche Maurin-A. Mortality curves observed in a few generations of Maurin-A strain.

La souche Maurin-A, créée à partir de la population naturelle Maurin-F0, est élevée sans pression de sélection au chlorpyrifos. La résistance se stabilise à partir de la F14. Les courbes (en pointillé) des mortalités des souches Bleuet et S54 servent de référence.

This strain was started from the Maurin-FO natural population and maintained without selection with chlorpyrifos. Resistance became stable from generation Fl4 on. Dotted lines represent the mortality curves of 554 and Bleuet strains. 
2. Évolution de la résistance dans la souche Maurin-A.

Dans cette souche, maintenue sans pression de sélection, la résistance a considérablement décru au cours des générations (fig. 2). Les courbes de mortalité restent globalement semblables entre elles au cours des générations et sont mal représentées par des droites de régression en raison de l'existence d'un plateau qui se maintient autour de 80 p. 100 de mortalité à partir de la $8^{\circ}$ génération. Dans la souche Maurin- $\mathrm{A}$, la $\mathrm{DL}_{50}$ est approximativement de $0,00075 \mathrm{ppm}$ depuis la $14^{\mathrm{e}}$ génération. Enfin, à la $38^{\circ}$ génération, aucun des 820 individus examinés ne possédaient le gène Est $-3^{A}$.

3. Evolution de la résistance dans la souche Maurin-B

La souche Maurin-B est maintenue en pression de sélection depuis sa fondation; la résistance y a considérablement augmenté : la $D_{50}$ de la $34^{\circ}$ génération était de 0,181 ppm de chlorpyrifos (intervalle de confiance : 0,176-0,185 ppm avec une probabilité d'erreur de 5 p. 100).

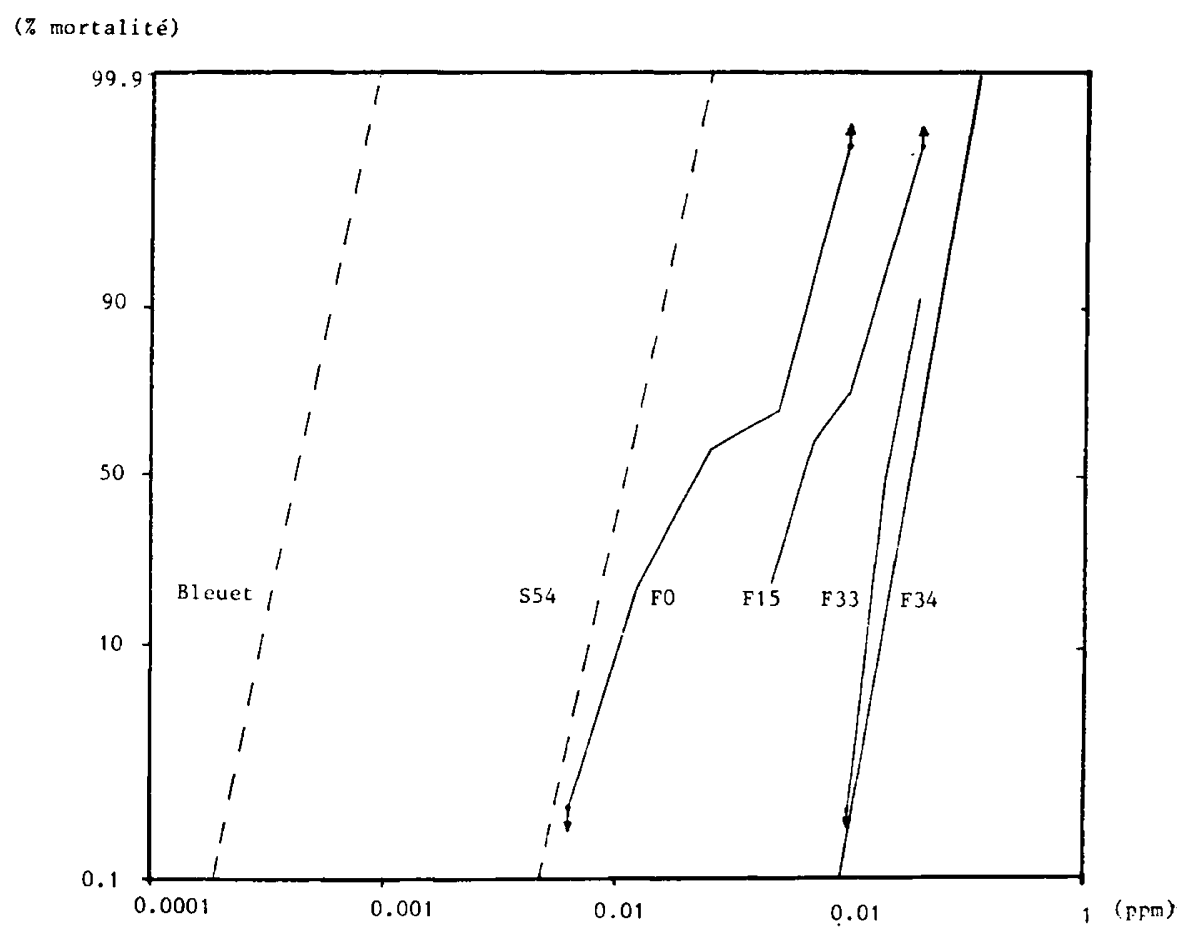

Fig. 3

Courbes de mortalité de quelques générations de la souche Maurin-B. Mortality curves observed in a few generations of Maurin-B strain.

La souche Maurin-B, créée à partir de la population naturelle Maurin-F0, est élevée en exerçant une pression de sélection au chlorpyrifos à chaque génération. Les courbes (en pointillé) des mortalités des souches Blcuet et $\mathbf{S} 54$ servent de référence.

This strain was started from the Maurin-Fo natural population by applying a selection with chlorpyrifos to larvae of each generation. Doted lines represent the mortality curves of S54 and Bleuet strains. 
L'augmentation de la résistance s'est effectuée de manière progressive (fig. 3) et se poursuit encore actuellement. Le plateau observé à la F0 disparaît rapidement et à la $34^{\mathrm{e}}$ génération, la courbe de mortalité est bien représentée par une droite de régression $(P=0,45)$ qui est parallèle aux droites de la souche résistante $\mathrm{S} 54$ et de la souche sensible Bleuet ( $\mathrm{P}=0,34$ et 0,972 respectivement).

L'étude de l'allèle Est-3A a révélé que 70 des 88 individus (soit 79,5 p. 100) de la $35^{\mathrm{e}}$ génération le possédaient. Ce gène, contrairement à ce qui s'est passé dans la souche Maurin-A, n'a donc pas disparu dans l'élevage soumis à la sélection.

\section{Evolution de la résistance dans la souche Maurin-C}

Issue de la F34 de la souche Maurin-B, la souche Maurin-C a été élevée sans pression de sélection. La perte de la résistance a été extrêmement rapide, la $\mathrm{DL}_{50}$ passant de $0,181 \mathrm{ppm}$ de chlorpyrifos à $0,006 \mathrm{ppm}$ en 5 générations seulement. Dès la $1^{\text {re }}$ génération sans sélection, nous avons constaté, dans la courbe de mortalité, l'apparition d'un léger plateau qui est rapidement devenu marqué (fig. 4). La fréquence de 1'allèle $E s t-3^{A}$ a été estimée chez les adultes de la $2^{\mathrm{e}}$ génération : il était présent chez 406 des 664 (soit 61,1 p. 100) individus étudiés. Ainsi, entre 2 générations la fréquence de l'allèle Est-3 ${ }^{A}$ a considérablement décru, et l'ampleur de cette perte ne semble pas corrélée avec celle du degré de résistance observé dans les tests insecticides. Cette observation nous a conduit 1) à rechercher la cause de la perte de l'allèle Est-3 ${ }^{A}$ dans Maurin-C, et 2) à isoler, à partir de Maurin-C, une nouvelle souche (MSE) qui ne possède pas l'allèle Est-3A .

\section{TABLEAU 3}

Phénotype des individus au locus Est-3 en fonction du jour d'émergence.

Observed and expected (in parenthesis) Est-3 phenotypes among adults emerged on different days.

\begin{tabular}{|c|c|c|c|c|}
\hline & $17-1-1983$ & $20-1-1983$ & 24-1-1983 & Total \\
\hline $\begin{array}{l}\text { Moustiques } \\
\text { avec le gène Est-3A }\end{array}$ & $117^{(141,2)}$ & $182(176,1)$ & 107 & 406 \\
\hline $\begin{array}{l}\text { Moustiques } \\
\text { sans le gène Est-3A }\end{array}$ & $114 \quad(89,8)$ & $106 \quad(111,9)$ & $(56,3)$ & 258 \\
\hline Total & 231 & 288 & 145 & 664 \\
\hline
\end{tabular}

Valeurs attendues entre parenthèse. Tous les moustiques proviennent d'œufs pondus la même nuit. Explication dans le texte.

All mosquitoes arise from eggs laid during the same night (see text).

$\underline{P}\left(X_{2}=20,98 ;\right.$ ddl $\left.=2\right)=0,999973$. 
(\% mortalité)

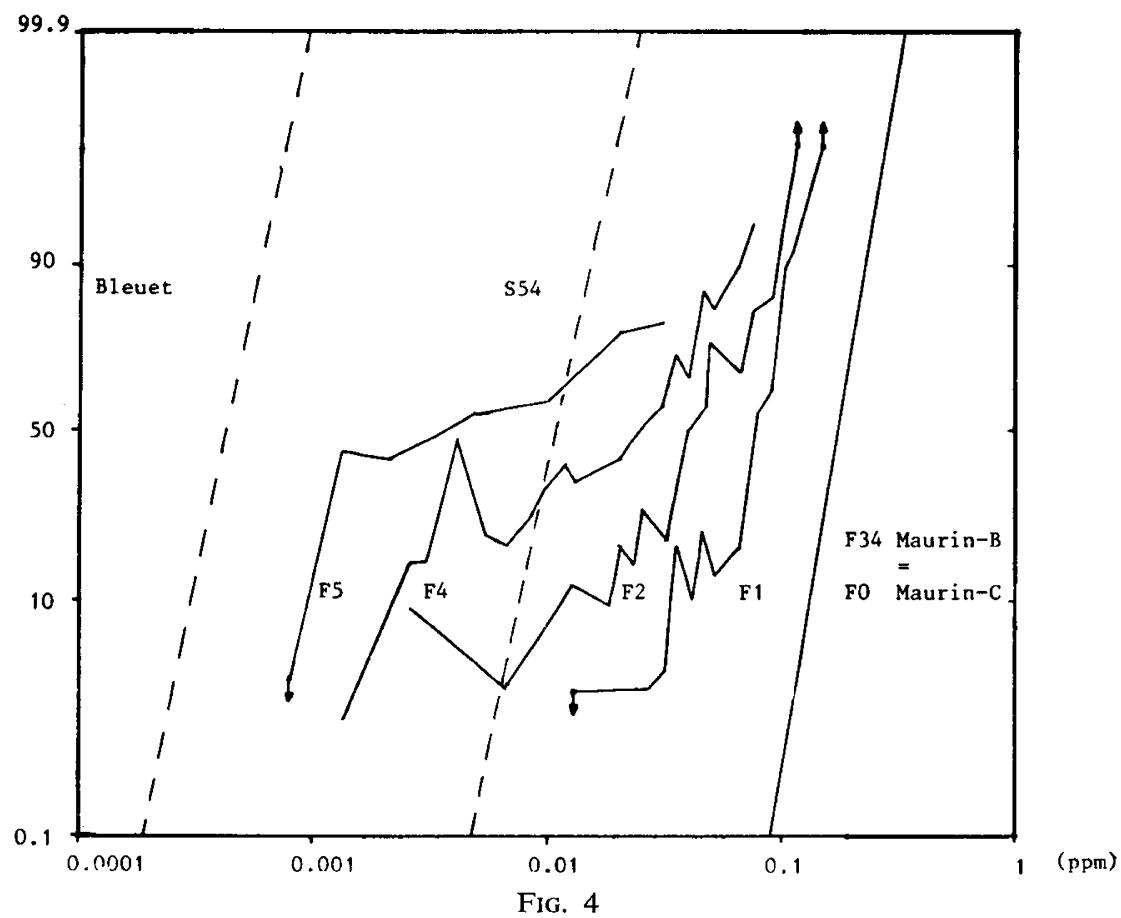

Courbes de mortalité de la souche Maurin-C.

Mortalité curves observed in the Maurin-C strain.

Créée à partir de la F34 de Maurin-B, Maurin-C est élevée sans pression de sélection. Un plateau apparaît vers le bas et monte rapidement. Les courbes (en pointillé) des souches Bleuet et S54 servent de référence.

This strain was started from generation F34 of the Maurin-B strain and raised without exposure to chlorpyrifos. A plateau appeared in the lower part of the mortality curves and rose rapidly. Dotted lines represent the mortality curves of 554 and Bleuet strains.

a) Origine de la perte de l'allèle $E s t-3^{A}$ dans la souche Maurin-C : sur la $2^{\mathrm{e}}$ génération d'élevage de la souche Maurin-C, nous avons recueilli 80 pontes produites dans la même nuit, et nous avons élevé une partie des larves qui en sont issues dans un même bac d'élevage. Les nymphes issues de ces larves ont été prélevées tous les jours et mises à éclore dans des cages différentes. Le phénotype du locus Est-3 a été déterminé pour tous les adultes éclos (tabl. 3). Il apparaît que parmi les adultes éclos pendant les premiers jours, 49,4 p. 100 ne possèdent pas l'allèle Est-3A alors que parmi ceux éclos les derniers jours 26,2 p. 100 ne l'ont pas. Les différences observées dans la fréquence des individus possédant ou ne possédant pas l'Estérase-3 en fonction du jour de nymphose (c'est-à-dire en fonction de la longeur du développement larvaire) sont hautement significatives $(P<0,0001)$. Ainsi, les individus homozygotes pour l'allèle Est-3O (qui ne possèdent pas l'Estérase-3) se développent plus rapidement que les individus homozygotes $E s t-3^{A} / E s t-3^{A}$ ou hétérozygotes $E s t-3^{A} / E s t 3^{O}$ (qui possèdent cette enzyme). 
(\% mortalité)

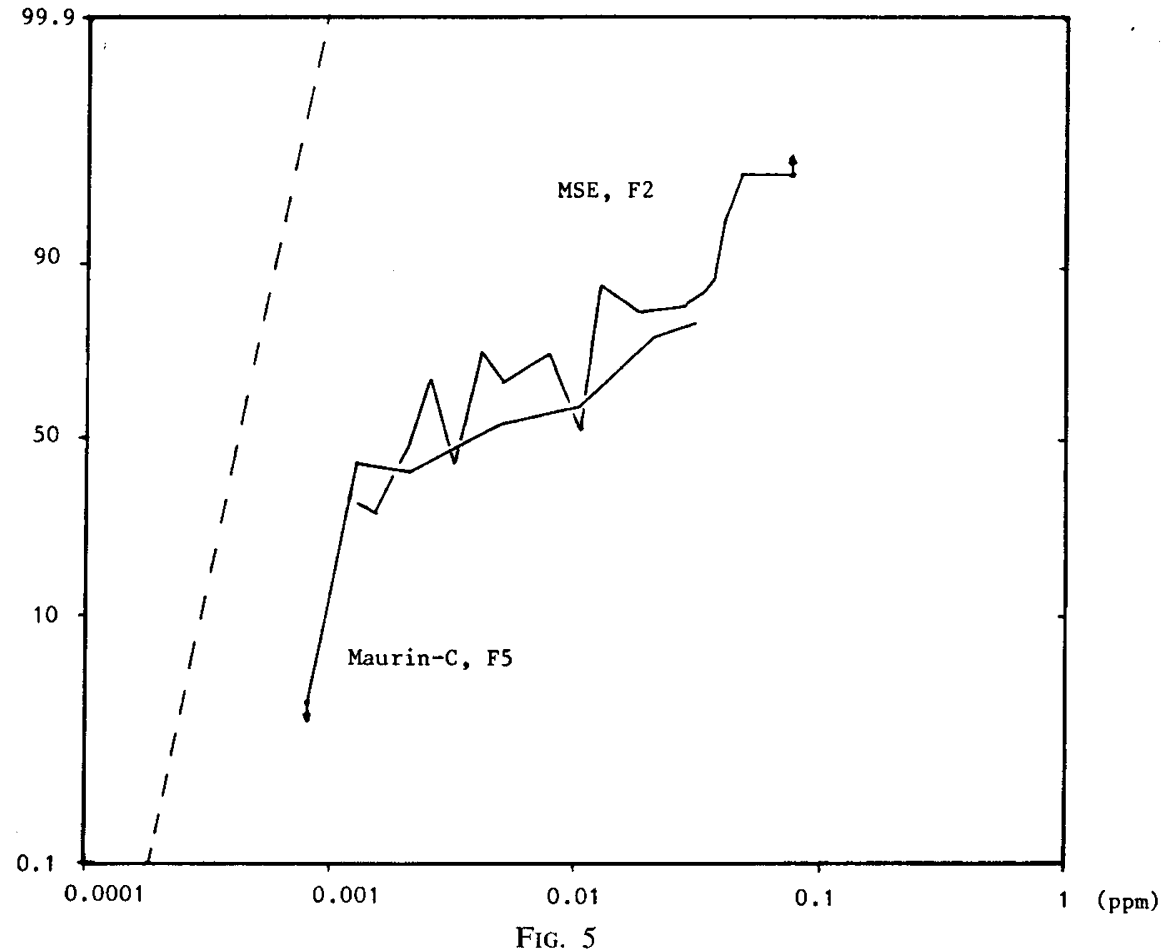

Comparaison de la F2 de MSE et de la F5 de Maurin-C.

Comparison of the mortality curves observed in the F2 generation of the MSE strain and in the $F 5$ generation of the Maurin $C$ strain.

La souche MSE est issue de la descendance des adultes de la $3^{\mathrm{c}}$ génération de Maurin-C qui ne possédaient pas l'allèle Est-3A. Ainsi, la génération F2 de MSE et F5 de Maurin-C sont comparables. Malgré l'absence de l'estérase de détoxification (l'allèle $E s t-3 A$ ), les 2 courbes de mortalité ont le même aspect. Souche sensible en pointillé.

The MSE strain was started from adults of the F3 generation of Maurin-C that lacked the Est-3A allele. Thus generations F2 of MSE and F5 of Maurin-C are comparable. Despite the absence of the detoxifying esterase (Est-3A allele) both mortality curves have the same aspect. Dotted line represents the mortality of the susceptible Bleuet strain.

b) Résistance de la souche MSE. Une partie des adultes de la $3^{\text {e }}$ génération de la souche Maurin-C a été isolée par couples, et la descendance des couples homozygotes pour 1'allèle $E s t-3^{O}$ (individus ne possédant pas 1'Estérase-3) a été utilisée pour fonder la souche MSE. L'absence de l'allèle $E s t-3^{A}$ a été vérifiée sur 124,53 et 248 adultes de $1 \mathrm{la} 1^{\mathrm{re}}, 2^{\mathrm{e}}$ et $4^{\mathrm{e}}$ génération d'élevage de cette nouvelle souche MSE. Les courbes de mortalité de Maurin-C et MSE de génération équivalente ont une allure générale assez semblable (fig. 5). Il semble donc que la présence de l'allèle Est-3A dans Maurin-C a peu d'influence sur la résistance globale. 


\section{Discussion}

Le contrôle des populations naturelles d'insectes nuisibles par les insecticides induit quasi invariablement l'apparition de résistances qui, au cours du temps, deviennent de plus en plus importantes.

Chez Culex pipiens, les populations de Californie présentent une résistance élevée à divers insecticides organophosphorés (RANASINGHE, 1976). Cette résistance est provoquée par l'allèle $E s t-2^{A}$ d'un gène unique responsable de la synthèse d'une estérase (Estérase-2) qui possède, comme 1'Estérase-3 observée dans les populations françaises, une grande activité vis-à-vis de l'alpha-naphthylacétate (GEORGHIOU \& PASTEUR, 1978 ; GEORGHIOU et al., 1980 ; PASTEUR et al., 1981 b). L'augmentation de résistance dans les populations de Californie (PASTEUR et al., 1980) est le résultat de l'apparition, au niveau du gène Est-2, d'allèles qui d'après PASTEur et al. (1984) correspondraient à des niveaux différents d'amplification (duplications répétées) de. l'allèle initial.

Les résultats que nous avons présentés ici indiquent que l'augmentation de la résistance aux organophosphorés dans les populations françaises de C. pipiens s'effectue par un mécanisme différent de celui qui prévaut dans les populations de Californie. En effet, nous avons montré, d'une part, qu'il n'est pas possible d'augmenter expérimentalement la résistance de la souche S54 homozygote pour l'allèle Est-3A, et, d'autre part, qu'il est possible, à partir d'une population naturelle, d'obtenir des souches résistantes ne possédant pas l'allèle $E s t-3^{A}$. Il apparaît donc que, dans le Sud de la France, l'augmentation de résistance aux organophosphates observée depuis 1977 est le résultat de la sélection d'un ou plusieurs facteurs de résistance distincts de l'allèle Est-3A.

Notons que l'existence de facteurs de résistance différents de Est-3A était soupçonnée. PASteur et al. (1981 a), dans une étude portant sur 43 populations naturelles. prélevées en 1978 dans le midi de la France, observaient que certaines populations, homozygotes pour l'allèle Est-3A, n'étaient pas du tout affectées par des doses tuant un peu plus de 50 p. 100 des moustiques de la souche S54. Wood et al. (1984) notaient que la souche Maurin-B $\left(25^{\mathrm{e}}\right.$ génération) est non seulement résistante aux organophosphates, mais aussi aux carbamates (Sevin), ce qui n'est pas le cas de S54.

\section{A. Nature du ou des facteurs de résistance}

Nos résultats montrent que l'allèle $E s t-3^{A}$ (seul responsable de la résistance dans la souche $\mathrm{S} 54$ ) participe relativement peu à la résistance globale des souches où le ou les facteurs de résistance sont présents :

1) il n'existe pas de corrélation évidente entre le niveau du plateau de la courbe de mortalité et la fréquence des individus sans Est $3^{A}$ dans la $2^{\circ}$ génération de la souche Maurin-C élevée sans pression de sélection (plateau à environ 10 p. $100 \mathrm{de}$ mortalité ; individus sans $E s t-3^{A}=38,8$ p. $100 \pm 3,7$ ) et, surtout,

2) l'aspect des courbes de mortalité de 2 lots de moustiques ( $2^{\mathbf{e}}$ génération de MSE ne possédant pas l'allèle Est-3A et $5^{\mathrm{e}}$ génération de Maurin-C composée d'un mélange d'individus avec et sans $E s t-3^{A}$ ) élevés depuis le même nombre de générations sans pression de sélection, est quasi identique (fig. 5). 
Ces observations suggèrent que l'efficacité du facteur de résistance est telle que, quand il est présent, l'action de l'allèle Est-3 ${ }^{A}$ devient secondaire ; une modification de la « cible » (i.e. acéthylcholinestérase des synapses) pourrait correspondre à ce cas et expliquerait en même temps l'existence d'une résistance aux carbamates dans la souche Maurin-B (AyAD \& GEorghiou, 1975). Mais on peut invoquer aussi des mécanismes de détoxification ou de perméabilité membranaire, agissant sur l'insecticide plus efficacement que l'Estérase-3.

\section{B. Valeurs adaptatives de l'allèle Est-3A et du facteur « Res»}

1) L'allèle $E s t-3^{A}$ n'est pas neutre quand on relâche la pression de sélection. L'absence de l'Estérase-3 dans la souche Maurin-A est sûrement le résultat d'une perte de l'allèle $E s t-3^{A}$ au cours des différentes générations. On peut effectivement considérer que cet allèle était présent dans la souche de départ, Maurin F0 (cf. ci-dessus) ; sa présence dans la souche sélectionnée, Maurin- $B$, conforte cette hypothèse. Nous avons vu par ailleurs (cf. F2 de.Maurin-C) que le fait de posséder au moins un allèle $\dot{E} s t-3^{A}$ entraînait un temps de développement larvaire plus long.

On peut donc penser que l'allèle $E s t-3^{A}$ (ou un autre allèle qui lui est associé) est contre-sélectionné en l'absence de chlorpyrifos. La méthode d'élevage utilisée, qui ne retient pas les dernières larves afin d'éviter que les générations se chevauchent, pourrait accélérer une telle sélection.

2) L'allèle $E s t-3^{A}$ n'est pas neutre quand on maintient la pression de sélection. Puisque Est-3A est moins efficace que le facteur « Res » et qu'il procure une valeur adaptative plus faible, il devrait avoir tendance à disparaître des populations, même quand celles-ci sont soumises aux insecticides. Or, dans la souche Maurin-B, Est-3A a été conservé à une fréquence importante. Ainsi, on est amené à supposer qu'en présence de chlorpyrifos, l'allèle $E s t-3^{A}$ présente un certain avantage puisqu'il est retenu. Ceci peut s'expliquer si l'on considère que de fortes concentrations d'insecticide pourraient saturer le facteur de résistance et ainsi favoriser les individus possédant par surcroît l'allèle Est-3 ${ }^{A}$.

Mais si l'allèle $E s t-3^{A}$ procure un avantage en présence de chlorpyrifos, comment se fait-il qu'il subsiste dans la souche Maurin-B au moins 20 p. 100 des individus ne possédant pas cet allèle?

3) Le facteur de résistance n'est pas neutre quand on relâche la pression de sélection. Le plateau qui apparaît vers le bas et qui monte, dans la souche Maurin-C, exprime une diminution en fréquence du facteur de résistance au cours des générations. Ce dernier est donc sûrement contre-sélectionné en l'absence de pression de sélection.

\section{Nature de l'évolution de la résistance dans les diverses souches au cours du temps}

Quand la résistance est monofactorielle, $R$ étant 1'allèle de résistance et $S$ l'allèle sensible correspondant, 3 types d'individus existent dans une population : SS, homozygotes sensibles, $R R$, homozygotes résistants et $R S$ hétérozygotes dont le comportement en présence d'insecticide est a) semblable à celui des $S S$ ou des $R R$ selon que 
(\% mortalité)

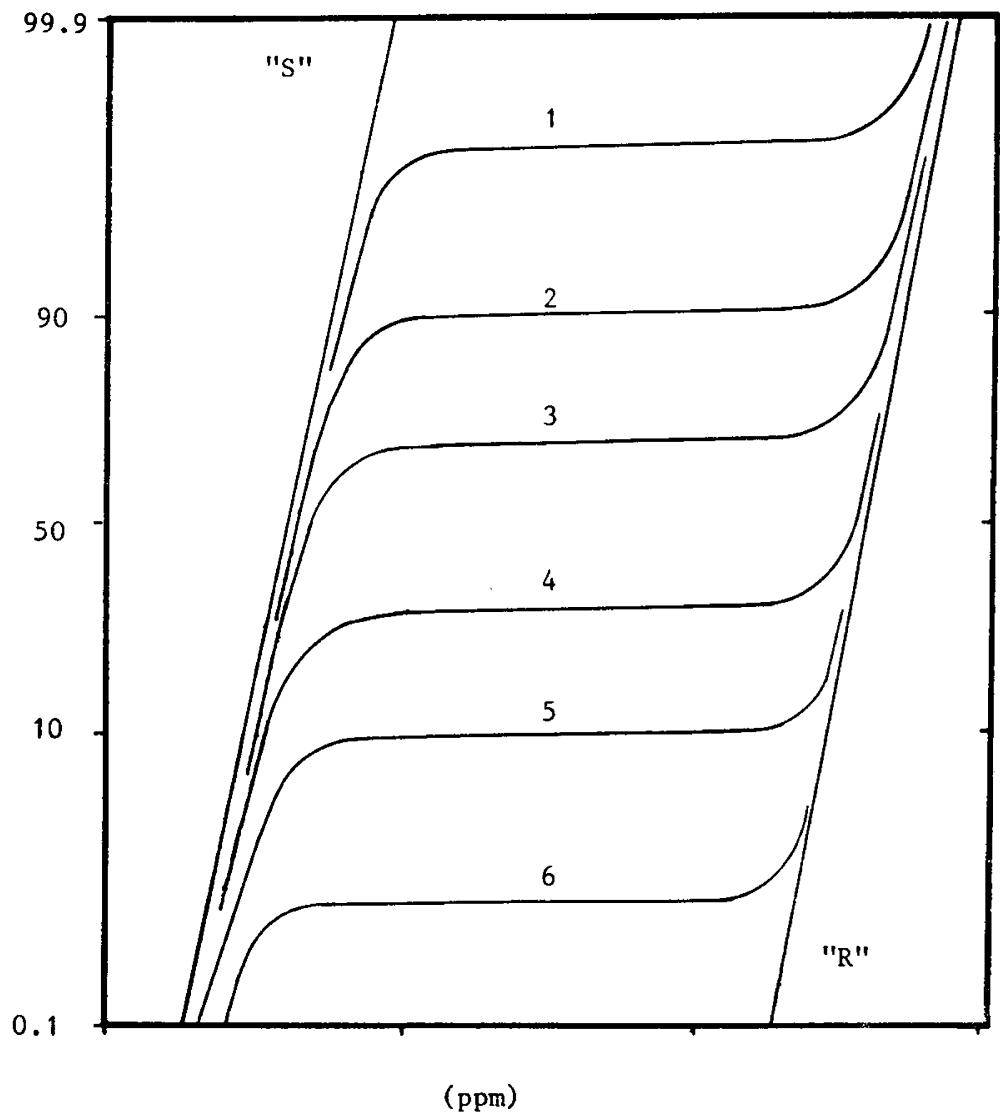

Fig. 6

Evolution théorique d'une courbe de mortalité en pression de sélection (sens 1-6) ou en relâchement de pression de sélection (sens 6-1) d'une population où la résistance est due à un gène unique.

Theoretical evolution of mortality curves obtained when selection pressure is applied (from 1 to 6 ) or relaxed (from 6 to 1 ) in a population in which resistance is controlled by a unique gene.

« $\mathrm{S}$ » : phénotype sensible correspondant aux homozygotes $S S$ et aux hétérozygotes $R S$. si l'allèle $\mathbf{R}$ est totalement récessif, « $\mathbf{R}$ »: phénotype résistant correspondant à des hétérozygotes $R S$ (si l'allèle $\mathrm{R}$ est partiellement dominant) ou à des génotypes $R R$ et $R S$ (si l'allèle $\mathbf{R}$ est totalement dominant).

« $S$ » susceptible phenotypes and « $R$ » resistant phenotypes. If the $S$ allele has: complete dominance, « $S$ » phenotypes represent RS and SS genotypes; if the $S$ allele is completly recessive, " $S$ » phenotypes correspond solely to SS genotypes and « $R$ » phenotypes to both $\mathrm{RS}$ and $\mathrm{RR}$ genotypes. If the $R$ allele is only partially dominant as often occurs, « $R$ » phenotypes represent RS genotypes and « $S$ » correspond to the SS genotypes. 
l'allèle $R$ est récessif ou dominant, respectivement ou b) intermédiaire si l'allèle $R$ est codominant. Ces 3 génotypes correspondent le plus généralement à 2 phénotypes « $S$ » et « $R$ ». Une population polymorphe au locus considéré présentera un plateau dans la courbe de mortalité. Le niveau de ce plateau correspond au pourcentage d'individus de phénotype « $S$ ». Quand la fréquence des individus de phénotype « $S$ » diminue, lors d'une exposition de la population à des doses d'insecticides adéquates, le plateau se déplace vers le bas et disparaît quand les individus de phénotypes « $R$ » sont seuls présents (fig. 6). Inversement une diminution du pourcentage des individus de phénotype « $\mathrm{R}$ » se traduit par une montée du plateau, puis par une disparition de ce dernier quand seuls restent les individus de phénotype " $S$ ».

Parmi les courbes de résistance obtenues au cours de générations successives sur les souches issues de la population naturelle prélevée à Maurin, seules celles de la souche Maurin-C (relâchement de la sélection après 34 générations) suit le schéma classique décrit ci-dessus. On peut donc conclure que dans cette souche, la résistance était très probablement en grande partie due à un gène majeur (qui masque l'action de l'allèle $E s t-3^{A}$ aussi présent dans la souche).

Quand la résistance est multifactorielle, elle devient un phénomène quantitatif. Une perte de résistance ne se manifeste pas par l'apparition et la montée d'un plateau, mais par une translation vers la droite de la courbe de mortalité qui correspond à la multiplicité des phénomènes de perte, et inversement lors d'un accroissement de la résistance. Remarquons que l'augmentation de l'amplification d'un gène de résistance donne le même type de déplacement des courbes de mortalité puisque les différents états amplifiés peuvent être assimilés aux différents gènes d'un système polygénique.

Pour les souches Maurin-A ( = population naturelle élevée sans pression de sélection, fig. 2) et Maurin-B (= population naturelle élevée en sélection, fig. 3), on constate que les courbes de résistance conservent à peu près la même forme au cours des générations et que l'on passe d'une génération à l'autre par une translation sans qu'apparaisse de plateau dans les zones de mortalité élevée (Maurin-B) ou basse (Maurin-A).

Il faut alors expliquer comment la résistance, apparemment multifactorielle dans les souches Maurin-A et Maurin-B est devenue monofactorielle dans la souche Maurin-C.

Nous ne pourrons avancer une hypothèse que quand nous aurons les résultats des croisements entre les diverses souches utilisées ici.

\section{Conclusions}

La situation simple de 1975, où, dans le sud de la France, un seul gène était tenu responsable de la résistance au chlorpyrifos, est actuellement plus complexe. Le ou les nouveaux facteurs, plus efficaces ,procurent aux populations qui le portent des résistances bien plus élevées $(\times 200)$ que celles données par l'allèle Est-3A $(\times 20)$. Des interactions entre ces 2 facteurs existent, puisque l'allèle $E s t-3^{A}$, bien que moins efficace et associé à un retard dans le développement larvaire, se maintient aussi bien 
dans les populations naturelles traitées que dans les souches soumises à des pressions de sélection. La nature et le mode d'action du ou des facteurs de résistance ne sont pas connues.

Reçu le 7 décembre 1983.

Accepté le 15 mai 1984.

\section{Références bibliographiques}

Axad H., Georghiou G.P., 1975. Resistance to organophosphates and carbamates in Anopheles albimanus based on reduced sensitivity of acethylcholinesterase. J. Econ. Entomol., 68, 295-297.

GeORGHIOU G.P., PASTEUR N., 1978. Electrophoretic patterns in insecticide-resistant and susceptible mosquitoes. J. Econ. Entomol., 71, 201-205.

Georghiou G.P., Pasteur N., HaWley M.K., 1980. Linkage relationships between organophosphate resistance and a highly active esterase $\mathrm{B}$ in Culex quinquefasciatus Say from California. J. Econ. Entomol., 73, 301-305.

Pasteur N., 1977. Recherches de génétique chez Culex pipiens pipiens L. Polymorphisme enzymatique, autogénèse et résistance aux insecticides organophosphorés, $170 \mathrm{pp}$., thèse doctorat d'Etat, U.S.T.L., Montpellier.

Pasteur N., Iseki A., Georghiou G.P., 1981 b. Genetic and biochemical studies of the highly active esterase $A^{\prime}$ and $B$ associated with organophosphate resistance in mosquitoes of the Culex pipiens complex. Biochem. Genet., 19, 499.508.

Pasteur N., Georghiou G.P., 1981. Filter paper test for rapid determination of phenotypes with high esterase activity in organophosphate resistant mosquitoes. Mosq. News, 41, 181-183.

Pasteur N., Georghiou G.P., IseKI A., 1984. Variation in organophosphate resistance and esterase activity in Culex quinquefasciatus Say from California. Génét. Sél. Evol., 16, 271-283.

Pasteur N., Georghiou G.P., Ranasinghe L.E., 1980. Variation in the degree of homozygous resistance to organophosphorus insecticides in Culex quinquefasciatus Say. Proceeding and Papers of the Forty-eight Annual Conference of the California Mosquito and Vector Control Association, Inc. Annaheim, January 20-23, 1980, 69-73, California Mosquito and Vector Control Association, Visalia, California, U.S.A.

PASTEUR N., Sinegre G., 1975. Esterase polymorphism and sensitivity to Dursban organophosphorus insecticide in Culex pipiens pipiens populations. Biochem. Genet., 13, 789-803.

Pasteur N., Sinegre G., 1978 a. Autogenesis vs. esterase polymorphism and chlorpyrifos (Dursban) resistance in Culex pipiens pipiens L. Biochem. Genet., 16, 941-943.

PASTEUR N., SINEgRe G., 1978 b. Chlorpyrifos (Dursban) resistance in Culex pipiens pipiens L. from southern France : inheritance and linkage. Experientia, 34, 709-711.

Pasteur N., Sinegre G., Gabinaud A., 1981 a. Est-2 and Est-3 polymorphisms in Culex pipiens L. from southern France in relation to organophosphate resistance. Biochem. Genet., 19, 499-508.

RANASINGHE L.E., 1976. Role of synergists in the selection of specific organophosphorus resistance mechanisms in Culex pipiens quinquefasciatus Say, 122 pp., Ph.D. dissertation, University of California, Riverside.

Rioux J.A., PECH J., 1961. Apparition de l'autogénèse dans un élevage de Culex pipiens berbericus Roubaud. C.R. Soc. Biol., 155, 343-344.

Sinegre G., Jullien J.L., Crespo O., 1976. Résistances de certaines populations de Culex pipiens (L.) au chlorpyrifos (Dursban) en Languedoc-Roussillon (France). Cahier O.R.S.T.O.M., Ser. Entomol. méd. et Parasitol., 14, 49-59.

Sinegre G., Jullien J.L., Gaven O., 1977. Acquisition progressive de la résistance au chlorpyrifos chez les larves de Culex pipiens $\mathrm{L}$. dans le midi de la France. Parassitologia, 19, 79-94.

Wood R., Pasteur N., Sinegre G., 1984. Carbamate and organophosphate resistance in the Culex pipiens complex in Southern France and the significance of esterase-3 A. Bull. Ent. Res., 74 (sous presse). 